\title{
A LOGIC WITH HIGHER ORDER CONDITIONAL PROBABILITIES
}

\section{Zoran Ognjanović and Nebojša Ikodinović}

\begin{abstract}
We investigate probability logic with the conditional probability operators. This logic, denoted $L C P$, allows making statements such as: $P_{\geqslant s} \alpha$, $C P_{\geqslant s}(\alpha \mid \beta), C P_{\leqslant 0}(\alpha \mid \beta)$ with the intended meaning "the probability of $\alpha$ is at least $s$ ", "the conditional probability of $\alpha$ given $\beta$ is at least $s$ ", "the conditional probability of $\alpha$ given $\beta$ at most 0 ". A possible-world approach is proposed to give semantics to such formulas. Every world of a given set of worlds is equipped with a probability space and conditional probability is derived in the usual way: $P(\alpha \mid \beta)=\frac{P(\alpha \wedge \beta)}{P(\beta)}, P(\beta)>0$, by the (unconditional) probability measure that is defined on an algebra of subsets of possible worlds. Infinitary axiomatic system for our logic which is sound and complete with respect to the mentioned class of models is given. Decidability of the presented logic is proved.
\end{abstract}

\section{Introduction}

The aim of probabilistic logics is clearly to capture the rules of reasoning about uncertain knowledge. One of the crucial issue in uncertain reasoning is the notion of conditional probability. Nilsson, for example, in a review [10] of work subsequent to his paper [9], argue that the conditional probability of $\beta$ given $\alpha$ reflects more accurately what we normally mean by the certainty of the rule 'if $\alpha$ then $\beta$ ', then the probability of $\alpha \rightarrow \beta$ does. In recent times, many authors stress the importance of conditional probabilities. Some of them even suggest to consider conditional probability and conditional events as basic notions, not derived from the notion of unconditional probability. This idea is actually quite old, but some formal treatments can be found in Popper $(1934,1938)$ and de Finetti $(1936,1949)$. The latter was the first who introduced the axioms for a direct definition of conditional probability (linking it to the concept of coherence, that allows to manage also 'partial' assessments). In [1] a rich elaboration of different issue of reasoning with the conditional probability, but only at semantical level, along the ideas proposed by de Finetti, is provided. In [6] we investigated a probability logic which

2000 Mathematics Subject Classification: Primary 68T37; Secondary 68T27, 03B48, 03B70.

Supported by Ministarstvo za nauku, tehnologije i razvoj Republike Srbije, through Matematički institut, under grant 1379. 
enriches propositional calculus with a class of conditional probability operators of de Finetti's type. But, allowing iterations of probability operators in that framework could produce some problems. In this paper we consider probability logic suitable for reasoning about conditional probability that is based on Kolmogorov's approach, allowing the iterations of probabilistic operators and mixing of classical and probabilistic formulas. Let's mention that iteration of probability and mixing classical and probabilistic formulas are not allowed in most of the logic for reasoning about conditional probabilities that may be found in the literature. Higher order absolute (unconditional) probabilities were allowed in the logic presented in [11].

The corresponding probability language is obtained by adding probability operators of the forms $P_{\geqslant s}, C P_{\geqslant s}$ and $C P_{\leqslant 0}$ to classical languages. It allows making formulas such as $P_{\geqslant s} \alpha$, with the intended meaning "the probability of truthfulness of $\alpha$ is greater than or equal to $s$ " and $C P_{\geqslant s}(\alpha \mid \beta)$, with the intended meaning "the conditional probability of truthfulness of $\alpha$ given $\beta$ is greater than or equal to $s$ ". The probability operators behave like modal operators. As the corresponding semantics we introduce special types of Kripke models with addition of probability measure defined over the words. It is well known that if we have a finitary axiomatization, then the compactness theorem: 'if every finite subset of a set $T$ of formulas is satisfiable, then $T$ is satisfiable' follows easily from the extended completeness theorem ('every set of formulas is satisfiable'). The compactness theorem does not hold for $L C P$. Namely, consider an arbitrary classical formula and the set $T=\left\{\neg P_{=0} \varphi\right\} \cup\left\{P_{<1 / n} \varphi: n\right.$ is a positive integer $\}$; although every finite subset of $T$ is satisfiable, the set $T$ itself is not. A consequence is that, if we want the extended completeness theorem, we cannot obtain a finitary axiomatization. Building on $[8,12,13,14,17]$, we define a system which we show to be sound and complete, using infinitary rules of inference (i.e., rules where a conclusion has a countable set of premises).

The rest of the paper is organized as follows. In Section 2 the syntax of the logic is given. Section 3 contains a description of the corresponding Kripke-style models and satisfiability and validity notions. A formulation of a sound and complete axiomatic system can be found in Section 4. Proofs of Soundness and Completeness theorems are presented in Section 5. The decidability is discussed in Section 6. We conclude in Section 7 with some remarks about similar approaches, open questions and further investigations.

\section{Syntax}

The language $\mathcal{L}$ of $L C P$ consists of a countable set $I=\left\{p_{1}, p_{2}, \ldots\right\}$ of propositional letters, classical connectives $\wedge$ and $\neg$, a list of unary probabilistic operators $P_{\geqslant s}$ and binary probability operators $C P_{\geqslant s}$ for every rational number $s \in[0,1]$, $C P_{\leqslant 0}$ and parentheses. The set For $_{L C P}$ of formulas is the smallest set of a finite sequences of symbols of the language $\mathcal{L}$ containing the propositional letters and closed under formation rules: if $\alpha$ and $\beta$ are formulas, then $\neg \alpha, P_{\geqslant} \alpha, C P_{\geqslant s}(\alpha \mid \beta)$, $C P_{\leqslant 0}(\alpha \mid \beta)$ and $\alpha \wedge \beta$ are formulas. For example, the following is a formula: $C P_{\geqslant 0.2}\left(p_{1} \rightarrow p_{2} \mid P_{\geqslant 0.12} P_{\geqslant 0.1} p_{1}\right)$. 
We use the usual abbreviations for the other classical connectives $\vee, \rightarrow, \leftrightarrow$. It is convenient the following abbreviations in our logic, for every rational number $s$ from $[0,1]$ :

and:

- $P_{<s}(\alpha) \stackrel{\text { def }}{=} \neg P_{\geqslant s}(\alpha), \quad$ - $P_{\leqslant s}(\alpha) \stackrel{\text { def }}{=} P_{\geqslant 1-s}(\neg \alpha)$,

- $P_{>s}(\alpha) \stackrel{\text { def }}{=} \neg P_{\leqslant s}(\alpha), \quad \bullet P_{=s}(\alpha) \stackrel{\text { def }}{=} P_{\geqslant s}(\alpha) \wedge P_{\leqslant s}(\alpha)$,

- $C P_{<s}(\alpha \mid \beta) \stackrel{\text { def }}{=} \neg C P_{\geqslant s}(\alpha \mid \beta)$,

- $C P_{\leqslant s}(\alpha \mid \beta) \stackrel{\text { def }}{=} C P_{\geqslant 1-s}(\neg \alpha \mid \beta)$, for $s \neq 0$,

- $C P_{>s}(\alpha \mid \beta) \stackrel{\text { def }}{=} \neg C P_{\leqslant s}(\alpha \mid \beta)$,

- $C P_{=s}(\alpha \mid \beta) \stackrel{\text { def }}{=} C P_{\geqslant s}(\alpha \mid \beta) \wedge C P_{\leqslant s}(\alpha \mid \beta)$.

Also, $\perp$ is used to denote $\theta \wedge \neg \theta$, for an arbitrary formula $\theta$. We use various conventions such as rules about deleting parenthesis and priority of logical connectives.

\section{Semantics}

The usual way of introducing probability is axiomatic through the measuretheoretic framework proposed by Kolmogorov.

A probability space $(\Omega, H, \mu)$ consists of

- a non-empty set $\Omega$ (called the sample space),

- an algebra $H$ of subsets of $\Omega$ containing $\Omega$ and closed under complementation and finite union (but not necessarily consisting of all subsets of $\Omega$ ), whose elements are called events (or measurable sets),

- a finitely additive measure $\mu: H \rightarrow[0,1]$ (called probability) satisfying:

$-\mu(\Omega)=1$,

- $\mu\left(A_{1} \cup A_{2}\right)=\mu\left(A_{1}\right)+\mu\left(A_{2}\right)$, for all disjoint sets $A_{1}, A_{2} \in H$.

Given a fixed $A$ with $\mu(A)>0$, probability measure $\mu(\cdot \mid A)$, called conditional probability, is defined as $\mu(B \mid A)=\mu(A \cap B) / \mu(A)$. The requirement $\mu(A)>0$ is essential. If $\mu(A)=0$, then the conditional probability remains undefined. Let's mention that Kolmogorov's approach has become so entrenched that it is often referred to as the definition of conditional probability.

We use the possible-worlds approach to give semantics to probabilistic formulas.

Definition 3.1. An $L C P$-model is a structure $\mathbf{M}=(W, v$, Prob $)$, where:

- $W$ is a non empty set of elements called worlds,

- $v: W \times I \rightarrow\{$ true, false $\}$ provides for each world $w \in W$ a two-valued evaluation of the propositional letters, that is $v(w, p) \in\{$ true, false $\}$, for each propositional letter $p \in I$ and each world $w \in W$; the truth-evaluation $v(w, \cdot)$ is extended to classical propositional formulas as usual,

- Prob assigns to every $w \in W$ a structure $(W(w), H(w), \mu(w))$, such that $W(w)$ is non empty subset of $W, H(w)$ is an algebra of subsets of $W(w)$ and $\mu(w): H(w) \rightarrow[0,1]$ is a finitely additive probability measure.

Definition 3.2. The satisfiability relation $\vDash_{\mathbf{M}} \subset W \times$ For $_{L C P}$ fulfills the following condition for every $L C P-\operatorname{model} \mathbf{M}=(W, v$, Prob) and every world $w \in W$ : 
- if $\varphi$ is a propositional letter, then: $w \vDash_{\mathbf{M}} \varphi$ iff $v(w, \varphi)=1$,

- if $\varphi=P_{\geqslant_{s}} \alpha$, then: $w \vDash_{\mathbf{M}} \varphi$ iff $\mu(w)\left(\left\{w^{\prime} \in W(w): w^{\prime} \vDash_{\mathbf{M}} \alpha\right\}\right) \geqslant s$,

- if $\varphi=C P_{\geqslant s}(\alpha \mid \beta)$, then: $w \vDash_{\mathbf{M}} \varphi$ iff either

$\mu(w)\left(\left\{w^{\prime} \in W(w): w^{\prime} \vDash_{\mathbf{M}} \beta\right\}\right)=0$ or $\mu(w)\left(\left\{w^{\prime} \in W(w): w^{\prime} \vDash_{\mathbf{M}} \beta\right\}\right)>0$

and

$$
\frac{\mu(w)\left(\left\{w^{\prime} \in W(w): w^{\prime} \vDash_{\mathbf{M}} \alpha\right\} \cap\left\{w^{\prime} \in W(w): w^{\prime} \vDash_{\mathbf{M}} \beta\right\}\right)}{\mu(w)\left(\left\{w^{\prime} \in W(w): w^{\prime} \vDash_{\mathbf{M}} \beta\right\}\right)} \geqslant s,
$$

- if $\varphi=C P_{\leqslant 0}(\alpha \mid \beta)$, then: $w \vDash_{\mathbf{M}} \varphi$ iff

$$
\mu(w)\left(\left\{w^{\prime} \in W(w): w^{\prime} \vDash_{\mathbf{M}} \alpha\right\} \cap\left\{w^{\prime} \in W(w): w^{\prime} \vDash_{\mathbf{M}} \beta\right\}\right)=0
$$

and

$$
\mu(w)\left(\left\{w^{\prime} \in W(w): w^{\prime} \vDash_{\mathbf{M}} \beta\right\}\right)>0,
$$

- if $\varphi=\alpha \wedge \beta$, then $w \vDash_{\mathbf{M}} \varphi$ iff $w \vDash_{\mathbf{M}} \alpha$ and $w \vDash_{\mathbf{M}} \beta$,

- if $\varphi=\neg \alpha$, then $w \vDash_{\mathbf{M}} \varphi$ iff not $w \vDash_{\mathbf{M}} \alpha$.

A formula $\varphi$ is satisfiable in a given $L C P$-model $\mathbf{M}$ if there is a world $w$ from $\mathbf{M}$ such that $w \vDash_{\mathbf{M}} \varphi$. A formula $\varphi$ is valid, if for every $L C P$-model $\mathbf{M}$ and each world $w$ from $\mathbf{M}, w \vDash_{\mathbf{M}} \varphi$.

An $L C P$-model $\mathbf{M}$ is measurable, if for every formula $\varphi$ and every world $w$ from $\mathbf{M},\left\{w^{\prime} \in W(w): w^{\prime} \vDash_{\mathbf{M}} \varphi\right\} \in H(w)$. We denote the class of all measurable models by $L C P_{\text {Meas }}$. In the sequel $[\varphi]_{\mathbf{M}, w}$ denotes $\left\{w^{\prime} \in W(w): w^{\prime} \vDash_{\mathbf{M}} \varphi\right\}$. We will omit the subscript $\mathbf{M}, w$ from $[\varphi]_{\mathbf{M}, w}$ and write $[\varphi]$ if $\mathbf{M}$ and $w$ are clear from the context.

If $\mu(w)\left([\beta]_{\mathbf{M}, w}\right)>0$, we will use $\mu(w)\left([\alpha]_{\mathbf{M}, w} \mid[\beta]_{\mathbf{M}, w}\right)$ to denote

$$
\frac{\mu(w)\left([\alpha]_{\mathbf{M}, w} \cap[\beta]_{\mathbf{M}, w}\right)}{\mu(w)\left([\beta]_{\mathbf{M}, w}\right)} .
$$

\section{Axiomatic system}

The axiomatic system $\mathrm{Ax}_{L C P}$ for $L C P$ contains the following axiom schemata:

(1) all For ${ }_{L C P}$-instances of classical propositional tautologies,

(2) $P_{\geqslant 0} \alpha$

(3) $P_{\leqslant r} \alpha \rightarrow P_{<s} \alpha, s>r$

(4) $P_{<s} \alpha \rightarrow P_{\leqslant s} \alpha$

(5) $\left(P_{\geqslant r} \alpha \wedge P_{\geqslant s} \beta \wedge P_{\geqslant 1}(\neg \alpha \vee \neg \beta)\right) \rightarrow P_{\geqslant \min (1, r+s)}(\alpha \vee \beta)$

(6) $\left(P_{\leqslant r} \alpha \wedge P_{<s} \beta\right) \rightarrow P_{<r+s}(\alpha \vee \beta), r+s \leqslant 1$

(7) $C P_{\geqslant s}(\alpha \mid \beta) \wedge P_{\geqslant t} \beta \rightarrow P_{\geqslant s \cdot t}(\alpha \wedge \beta), t>0$,

(8) $P_{=0}(\alpha \wedge \beta) \wedge P_{>0} \beta \leftrightarrow C P_{\leqslant 0}(\alpha \mid \beta)$,

and inference rules:

(1) From $\alpha$ and $\alpha \rightarrow \beta$ infer $\beta$.

(2) From $\alpha$ infer $P_{\geqslant 1} \alpha$.

(3) From $\beta \rightarrow P_{\geqslant s-\frac{1}{k}} \alpha$, for every integer $k \geqslant \frac{1}{s}$, infer $\beta \rightarrow P_{\geqslant s} \alpha$.

(4) From $\gamma \rightarrow\left(P_{\geqslant r} \beta \rightarrow P_{\geqslant r . s}(\alpha \wedge \beta)\right)$, for every rational number $r$ from $(0,1)$, infer $\gamma \rightarrow C P_{\geqslant s}(\alpha \mid \beta)$. 
The above axiomatic system is extension of the axiomatic system for the unconditional probability logic analyzed in $[\mathbf{1 1}, \mathbf{1 2}]$. The new axioms 7 and 8 and the inference rule 4 express the standard definition of the conditional probability. The infinitary inference rule 4 links, in the way to be expected, unary and binary probability operators

Definition 4.1. A formula $\alpha$ is a theorem $(\vdash \alpha)$ if there is an at most countable sequence of formulas $\alpha_{0}, \alpha_{1}, \ldots, \alpha$, such that every $\alpha_{i}$ is an axiom or it is derived from the preceding formulas of the sequence by an inference rule. In this paper we will also use the notion of deducibility. A formula $\alpha$ is deducible from a set $T$ of sentences $(T \vdash \alpha)$ if there is an at most countable sequence of formulas $\alpha_{0}, \alpha_{1}, \ldots, \alpha$, such that every $\alpha_{i}$ is an axiom or a formula from the set $T$, or it is derived from the preceding formulas by an inference rule, with the exception that the inference rule 2 can be applied on the theorems only. A set $T$ of sentences is consistent if there is at least one formula which is not deducible from $T$, otherwise $T$ is inconsistent. A consistent set $T$ of formulas is said to be maximal consistent if for every formula $\alpha$, either $\alpha \in T$ or $\neg \alpha \in T$.

The next theorem gives some auxiliary statements.

Theorem 4.1. (1) (Deduction theorem) If $T$ is a set of formulas, $\alpha$ is a formula, and $T \cup\{\alpha\} \vdash \beta$, then $T \vdash \alpha \rightarrow \beta$.

(2) Let $\alpha, \beta, \gamma$ be formulas. Then:

(a) $\vdash P_{\geqslant 1}(\alpha \rightarrow \beta) \rightarrow\left(P_{\geqslant_{s}} \alpha \rightarrow P_{\geqslant_{s}} \beta\right)$

(b) $\vdash P_{\geqslant r} \alpha \rightarrow P_{\geqslant s} \alpha, r>s$

(c) $\vdash C P_{\geqslant 0}(\alpha \mid \beta)$

(d) $\vdash C P_{\leqslant 1}(\alpha \mid \beta)$

(e) $\vdash C P_{\geqslant 1}(\alpha \mid \beta) \rightarrow C P_{=1}(\alpha \mid \beta)$

(f) $\vdash C P_{\geqslant 1}(\alpha \mid \beta) \rightarrow C P_{\geqslant s}(\alpha \mid \beta)$.

(g) if $\vdash \alpha \rightarrow \beta$, then $\vdash C P \geqslant 1(\beta \mid \alpha)$,

(h) $\vdash P_{=0} \beta \rightarrow C P_{=1}(\alpha \mid \beta)$.

Proof. 1. We use the transfinite induction on the length of the proof of $\beta$ from $T \cup\{\alpha\}$. The classical cases follow as usual.

Suppose that $\beta=\gamma \rightarrow C P_{\geqslant s}(\varphi \mid \psi)$ is obtained from $T \cup\{\alpha\}$ by an application of the inference rule 4 . Then:

$T, \alpha \vdash \gamma \rightarrow\left(P_{\geqslant r} \psi \rightarrow P_{\geqslant r . s}(\alpha \wedge \beta)\right)$, for all rational $r$ from $(0,1)$,

$T \vdash \alpha \rightarrow\left(\gamma \rightarrow\left(P_{\geqslant r} \psi \rightarrow P_{\geqslant r \cdot s}(\alpha \wedge \beta)\right)\right)$, for all $r$, by the induction hypothesis,

$T \vdash(\alpha \wedge \gamma) \rightarrow\left(P_{\geqslant r} \psi \rightarrow P_{\geqslant r \cdot s}(\alpha \wedge \beta)\right)$, for all $r$

$T \vdash(\alpha \wedge \gamma) \rightarrow C P_{\geqslant s}(\varphi \mid \psi)$, by the inference rule 4

$T \vdash \alpha \rightarrow \beta$.

The other cases follow similarly.

2. We prove some of the statements, while the other can be shown in a similar way $[\mathbf{1 3}, \mathbf{1 5}]$.

(4.1) By Axiom 7, we have $C P_{\geqslant 1}(\alpha \mid \beta) \vdash C P_{\geqslant 1}(\alpha \mid \beta) \wedge P_{\geqslant t} \beta \rightarrow P_{\geqslant t}(\alpha \wedge \beta)$, for all $t>0$, i.e. $C P_{\geqslant 1}(\alpha \mid \beta) \vdash P_{\geqslant t} \beta \rightarrow P_{\geqslant t}(\alpha \wedge \beta)$, for all $t$. Then, by 4.1 , we have 
$P_{\geqslant t}(\alpha \wedge \beta) \rightarrow P_{\geqslant t \cdot s}(\alpha \wedge \beta)$, and hence $C P_{\geqslant 1}(\alpha \mid \beta) \vdash P_{\geqslant t} \beta \rightarrow P_{\geqslant t \cdot s}(\alpha \wedge \beta)$, for all $t$. An application the inference rule 4 gives $C P_{\geqslant 1}(\alpha \mid \beta) \vdash C P_{\geqslant_{s}}(\alpha \mid \beta)$, i.e. $\vdash$ $C P_{\geqslant 1}(\alpha \mid \beta) \rightarrow C P_{\geqslant s}(\alpha \mid \beta)$, by Deduction theorem.

(4.1) Let $\vdash \alpha \rightarrow \beta$. Then $\vdash \alpha \rightarrow(\alpha \wedge \beta)$ and we have $\vdash P_{\geqslant 1}(\alpha \rightarrow(\alpha \wedge \beta))$, by inference rule 2. From (4.1), $\vdash P_{\geqslant r} \alpha \rightarrow P_{\geqslant r .1}(\alpha \wedge \beta)$, for every rational $r \in[0,1]$. Now, by inference rule $4(s=1)$, we obtain $\vdash C P_{\geqslant 1}(\beta \mid \alpha)$.

(4.1) Axioms 1-6 and Rules 1-3 imply: $\vdash P_{=0} \beta \rightarrow P_{=0}(\alpha \wedge \beta)$ and $\vdash P_{=0} \beta \rightarrow$ $\neg P_{\geqslant r} \beta$, for every rational $r \in(0,1]$. Then, for $s=1$ and every $r \in[0,1]$ we have $\vdash P_{=0} \beta \rightarrow\left(P_{\geqslant r} \beta \rightarrow P_{\geqslant r}(\alpha \wedge \beta)\right), \vdash P_{=0} \beta \rightarrow C P_{=1}(\alpha \mid \beta)$, by Rule 4 .

\section{Soundness and Completeness}

TheOREM 5.1 (Soundness theorem). The axiomatic system $A x_{L C P}$ is sound with respect to $L C P_{\text {Meas }}$ class of models.

Proof. Soundness of our system follows from the soundness of propositional classical logics and from the properties of probability, so we give only a sketch of a straightforward but tedious proof. We can show that every instance of an axiom schemata holds in every world of every $L C P_{\text {Meas }}$-model, while the inference rules preserve validity.

It is easy to see that if $\alpha$ is an instance of a classical propositional tautologies, then for every model $\mathbf{M}$ and each world from $\mathbf{M}, w \vDash_{\mathbf{M}} \alpha$.

Axioms 2-8 concern to the properties of measures and obviously holds in every world of a model. For example, let us consider Axiom 6. Let $\mathbf{M}$ be an $L C P$ model and $w$ an arbitrary world from $\mathbf{M}$. Then, from $[\alpha]_{\mathbf{M}, w}=\left([\alpha]_{\mathbf{M}, w} \cap(W(w) \backslash\right.$ $\left.[\beta]_{\mathbf{M}, w}\right) \cup[\alpha]_{\mathbf{M}, w}$, we have $\mu(w)\left([\alpha]_{\mathbf{M}, w}\right) \geqslant \mu(w)\left([\alpha]_{\mathbf{M}, w} \cap\left(W(w) \backslash[\beta]_{\mathbf{M}, w}\right)\right)$, for arbitrary $\alpha$ and $\beta$. From $[\alpha \vee \beta]_{\mathbf{M}, w}=\left([\alpha]_{\mathbf{M}, w} \cap\left(W(w) \backslash[\beta]_{\mathbf{M}, w}\right)\right) \cup[\beta]_{\mathbf{M}, w}$ it follows that $\mu(w)\left([\alpha \vee \beta]_{\mathbf{M}, w}\right) \leqslant \mu(w)\left([\alpha]_{\mathbf{M}, w}\right)+\mu(w)\left([\beta]_{\mathbf{M}, w}\right)$, i.e., Axiom 6 is valid. Similarly, from $\mu(w)\left([\alpha]_{\mathbf{M}, w} \mid[\beta]_{\mathbf{M}, w}\right) \cdot \mu(w)\left([\beta]_{\mathbf{M}, w}\right)=\mu(w)\left([\alpha]_{\mathbf{M}, w} \cap[\beta]_{\mathbf{M}, w}\right)$, $\mu(w)\left([\beta]_{\mathbf{M}, w}\right)>0$, follows that the axioms 7 and 8 are valid. The other axioms can be proved to be valid in a similar way.

Rule 1 is validity-preserving for the same reason as in classical logic. Consider Rule 2 and suppose that a formula $\alpha$ is valid. Then, for every world $w$ from a model $\mathbf{M}=\langle W, v, \operatorname{Prob}\rangle,[\alpha]_{\mathbf{M}, w}=W(w)$ and $\mu(w)(W(w))=1$. Hence, $P_{\geqslant 1} \alpha$ is valid too.

Rule 3 preserves validity because of the properties of the set of rational numbers.

Let us consider Rule 4 . Let $\mathbf{M}=\langle W, v$, Prob $\rangle$ be an $L C P_{\text {Meas-model, and }}$ $w \in W$ such that $w \vDash_{\mathbf{M}} A \rightarrow\left(P_{\geqslant r} \beta \rightarrow P_{\geqslant r \cdot s}(\alpha \wedge \beta)\right)$, for every rational number $r \in[0,1]$. Let $w \vDash_{\mathbf{M}} A$. If $\mu(w)([\beta])=0$, then $w \vDash_{\mathbf{M}} C P_{\geqslant_{1}}(\alpha \mid \beta)$, and $w \vDash_{\mathbf{M}} A \rightarrow$ $C P_{\geqslant s}(\alpha \mid \beta)$. Now, let $\mu(w)([\beta])=r_{0}>0$. Since the set of premisses holds in $w$, and $w \vDash_{\mathbf{M}} P_{\geqslant r} \beta$, for every $r \leqslant r_{0}$, it must be $\mu(w)([\alpha] \cap[\beta]) \geqslant r \cdot s$ (for $\left.r \leqslant r_{0}\right)$. If $\mu(w)([\alpha] \mid[\beta]) \geqslant s$, we have $\mu(w)([\alpha] \cap[\beta])=\mu(w)([\alpha],[\beta]) \cdot \mu(w)([\beta])<s \cdot r_{0}$, i.e. $w \not \nvdash_{\mathbf{M}} P_{\geqslant s \cdot r_{0}}(\alpha \wedge \beta)$. Thus, $\mu(w)([\alpha] \mid[\beta]) \geqslant s$, and $w \vDash_{\mathbf{M}} C P_{\geqslant s}(\alpha \mid \beta)$.

In order to prove the completeness theorems for our logics, we show that every consistent set of sentences is satisfiable. We describe how a consistent set $T$ of sentences can be extended to a suitable maximal consistent set, and how a canonical 
model can be constructed out of such maximal consistent sets. Finally, we prove that for every world $w$ from the canonical model, a sentence $\alpha$ is satisfied in $w$ if and only if $\alpha \in w$, and as a consequence we obtain that the set $T$ is satisfiable.

THEOREM 5.2. Every consistent set of formulas can be extended to a maximal consistent set.

Proof. Let $T$ be a consistent set of formulas and let $\alpha_{0}, \alpha_{1}, \ldots$ be an enumeration of all formulas. We define a sequence of sets $T_{i}, i=0,1,2, \ldots$ such that:

(1) $T_{0}=T$,

(2) for every $i \geqslant 0$, if $T_{i} \cup\left\{\alpha_{i}\right\}$ is consistent, then $T_{i+1}=T_{i} \cup\left\{\alpha_{i}\right\}$; otherwise $T_{i+1}=T_{i} \cup\left\{\neg \alpha_{i}\right\}$.

(3) if $T_{i+1}$ is obtained by adding a formula of the form $\neg\left(\beta \rightarrow P_{\geqslant s} \gamma\right)$, then for some positive integer $n, \beta \rightarrow \neg P_{\geqslant s-\frac{1}{n}} \gamma$, is also added to $T_{i+1}$, so that $T_{i+1}$ is consistent,

(4) if $T_{i+1}$ is obtained by adding a formula of the form $\neg\left(\beta \rightarrow C P_{\geqslant s}(\gamma \mid \delta)\right)$, then for some rational number $r^{\prime} \in(0,1), \beta \rightarrow \neg\left(P_{\geqslant r^{\prime}} \delta \rightarrow P_{\geqslant r^{\prime} \cdot s}(\gamma \wedge \delta)\right)$, is also added to $T_{i+1}$, so that $T_{i+1}$ is consistent.

The set obtained by the step 1 is obviously consistent. The step 2 produces consistent sets, too. For if $T_{i}, \alpha_{i} \vdash \perp$, by the deduction theorem we have $T_{i} \vdash \neg \alpha_{i}$, and since $T_{i}$ is consistent, so it is $T_{i} \cup\left\{\neg \alpha_{i}\right\}$. Consider the step 3. If $T_{i} \cup\left\{\beta \rightarrow P_{\geqslant_{s}} \gamma\right\}$ is not consistent, then the set $T_{i}$ can be consistently extended as it is described above. Suppose that it is not the case. Then:

(1) $T_{i}, \neg\left(\beta \rightarrow P_{\geqslant s} \gamma\right), \beta \rightarrow \neg P_{\geqslant s-\frac{1}{k}} \gamma \vdash \perp$, for every $k>\frac{1}{s}$, by the hypothesis

(2) $T_{i}, \neg\left(\beta \rightarrow P_{\geqslant s} \gamma\right) \vdash \neg\left(\beta \rightarrow \neg P_{\geqslant s-\frac{1}{k}} \gamma\right)$ for every $k>\frac{1}{s}$, by the deduction theorem

(3) $T_{i}, \neg\left(\beta \rightarrow P_{\geqslant s} \gamma\right) \vdash \beta \rightarrow P_{\geqslant s-\frac{1}{k}} \gamma$ for every $k>\frac{1}{s}$, from 2 ., by the classical tautology $\neg(\alpha \rightarrow \gamma) \rightarrow(\alpha \rightarrow \neg \gamma)$

(4) $T_{i}, \neg\left(\beta \rightarrow P_{\geqslant_{s}} \gamma\right) \vdash \beta \rightarrow P_{\geqslant s} \gamma$, from 3 , by the inference rule 3

(5) $T_{i} \vdash \neg\left(\beta \rightarrow P_{\geqslant_{s}} \gamma\right) \rightarrow \beta \rightarrow P_{\geqslant_{s}} \gamma$, from 4 , by Deduction theorem

(6) $T_{i} \vdash \beta \rightarrow P_{\geqslant s} \gamma$

Since $T_{i} \cup\left\{\beta \rightarrow P_{\geqslant s} \gamma\right\}$ is not consistent, from $T_{i} \vdash \beta \rightarrow P_{\geqslant_{s}} \gamma$ it follows that $T_{i}$ is not consistent, a contradiction. Thus, the step 3 produces consistent sets. Finally, consider the step 4 of the construction. If the set $T_{i} \cup\left\{\neg\left(\beta \rightarrow C P_{\geqslant_{s}}(\gamma \mid \delta)\right)\right\}$, is not consistent, then the set $T_{i}$ can be consistently extended as it is described above. Suppose that it is not the case. Then:

$T_{i}, \neg\left(\beta \rightarrow C P_{\geqslant s}(\gamma \mid \delta)\right), \beta \rightarrow \neg\left(P_{\geqslant r} \delta \rightarrow P_{\geqslant r \cdot s}(\gamma \wedge \delta)\right) \vdash \perp$, for all $r$ $T_{i}, \neg\left(\beta \rightarrow C P_{\geqslant s}(\gamma \mid \delta)\right) \vdash\left(\beta \rightarrow \neg\left(P_{\geqslant r} \delta \rightarrow P_{\geqslant r \cdot s}(\gamma \wedge \delta)\right)\right) \rightarrow \perp$, for all $r$, $T_{i}, \neg\left(\beta \rightarrow C P_{\geqslant s}(\gamma \mid \delta)\right) \vdash \neg\left(\beta \rightarrow \neg\left(P_{\geqslant r} \delta \rightarrow P_{\geqslant r \cdot s}(\gamma \wedge \delta)\right)\right)$, for all $r$, by the classical tautology $\neg(A \rightarrow B) \rightarrow(A \rightarrow \neg B)$, $T_{i}, \neg\left(\beta \rightarrow C P_{\geqslant s}(\gamma \mid \delta)\right) \vdash \beta \rightarrow\left(P_{\geqslant r} \delta \rightarrow P_{\geqslant r \cdot s}(\gamma \wedge \delta)\right)$, for all $r$ $T_{i}, \neg\left(\beta \rightarrow C P_{\geqslant s}(\gamma \mid \delta)\right) \vdash \beta \rightarrow C P_{\geqslant s}(\gamma \mid \delta)$, by the inference rule 4 .

Thus, $T_{i}$ is not consistent, a contradiction; hence, the step 4 produces consistent sets. 
Let $\bar{T}=\cup_{i} T_{i}$. We can show that $\bar{T}$ is a deductively closed set which does not contain all formulas, and as a consequence that $\bar{T}$ is consistent. First note that for every sentence $\alpha$, if $T_{i} \vdash \alpha$, then it must be $\alpha \in \bar{T}$. For if $\alpha=\alpha_{k}$, and $\alpha \notin \bar{T}$, then $T_{\max \{i, k\}+1} \vdash \alpha$ and $T_{\max \{i, k\}+1} \vdash \neg \alpha$, a contradiction. Let $\alpha$ be a sentence, and $\bar{T} \vdash \alpha$. If the deduction of $\alpha$ from $\bar{T}$ is a finite sequence, then there is some $i \geqslant 0$ such that $T_{i} \vdash \alpha$, and $\alpha \in \bar{T}$. Suppose that the sequence $\beta_{1}, \beta_{2}, \ldots, \alpha$ of formulas which forms the proof of $\alpha$ from $\bar{T}$ is countably infinite. We can show that for every $i$, if $\beta_{i}$ is obtained by an application of an inference rule, and all the premises of $\beta_{i}$ belong to $\bar{T}$, then $\beta_{i} \in \bar{T}$. Suppose $\beta_{i}$ is obtained by the inference rule 1 and its premises $\beta_{i}^{1}$ and $\beta_{i}^{2}$ belong to $\bar{T}$. There must be some $k$ such that $\beta_{i}^{1}, \beta_{i}^{2} \in T_{k}$. Since $T_{k} \vdash \beta_{i}$, it must be $\beta_{i} \in \bar{T}$. If $\beta_{i}$ is obtained by the inference rule 2 , then $\beta_{i}$ is a theorem and it must be $\beta_{i} \in \bar{T}$. If it is not, then $\alpha_{k}=\neg \beta_{i} \in T_{k+1}$, and $T_{k+1}$ is not consistent. Suppose that $\beta_{i}=\beta \rightarrow P_{\geqslant s} \gamma$ is obtained by the infinitary inference rule 3 , and that the premises $\beta_{i}^{1}=\beta \rightarrow P_{\geqslant s-\frac{1}{k}} \gamma, \beta_{i}^{2}=\beta \rightarrow P_{\geqslant s-\frac{1}{k+1}} \gamma, \ldots$ belong to $\bar{T}$. If $\beta \rightarrow P_{\geqslant s} \notin \bar{T}$, by the step 3 of the construction of $\bar{T}$, there is a $j>\frac{1}{s}$, such that $\beta \rightarrow \neg P_{\geqslant s-\frac{1}{j}} \gamma \in \bar{T}$. Let $l=\max \{k, j\}$. By the axioms 2 and 3, $\beta \rightarrow P_{\geqslant s-\frac{1}{l}} \gamma \in \bar{T}$, and $\beta \rightarrow \neg P_{\geqslant s-\frac{1}{l}} \gamma \in \bar{T}$. There is a set $T_{m}$ which also contains these formulas. It follows that $T_{m} \cup\{\beta\}$ is not consistent, and $\beta \notin \bar{T}$. There is some $j$ such that $\neg \beta \in T_{j}, T_{j} \vdash \beta \rightarrow \perp, T_{j} \vdash \beta \rightarrow P_{\geqslant s} \gamma$, and $\beta \rightarrow P_{\geqslant s} \gamma \in \bar{T}$, a contradiction. Finally, suppose that $\beta_{i}=\gamma \rightarrow C P_{\geqslant_{s}}(\varphi \mid \psi)$ is obtained by the infinitary inference rule 4 , and that the premises $\beta_{i}^{j}=\gamma \rightarrow\left(P_{\geqslant r_{j}} \psi \rightarrow P_{\geqslant r_{j}} \cdot s(\varphi \wedge \psi)\right)$ belong to $\bar{T}\left(r_{1}, r_{2}, \ldots\right.$ is an enumeration of all rational numbers from $\left.(0,1)\right)$. If $\gamma \rightarrow C P_{\geqslant s}(\varphi \mid \psi) \notin \bar{T}$, by the construction of $\bar{T}$, there is a $m$, such that $\gamma \rightarrow \neg\left(P_{\geqslant r_{j}} \psi \rightarrow P_{\geqslant r_{j} \cdot s}(\varphi \wedge \psi)\right) \in \bar{T}$. There is a set $T_{k}$ which contains formulas $\gamma \rightarrow\left(P_{\geqslant r_{j}} \psi \rightarrow P_{\geqslant r_{j} \cdot s}(\varphi \wedge \psi)\right)$ and $\gamma \rightarrow \neg\left(P_{\geqslant r_{j}} \psi \rightarrow P_{\geqslant r_{j} \cdot s}(\varphi \wedge \psi)\right)$. It follows that $T_{k} \cup\{\gamma\}$ is not consistent, and $\gamma \notin \bar{T}$. Then there is $l$ such that $\neg \gamma \in T_{l}, T_{l} \vdash \gamma \rightarrow \perp$ and $T_{l} \vdash \gamma \rightarrow C P_{\geqslant s}(\varphi \mid \psi)$, a contradiction. Thus, the set $\bar{T}$ is deductively closed. It does not contain all formulas. If for some $\alpha$, both $\alpha$ and $\neg \alpha$ belong to $\bar{T}$, then there is a set $T_{i}$ such that $\alpha, \neg \alpha \in T_{i}$, a contradiction because every $T_{i}$ is consistent. Thus, $\bar{T}$ is consistent.

From the step 2 of the construction, it follows that the set $\bar{T}$ is maximal.

The next theorem summarizes some obvious properties of the maximal consistent sets of formulas.

ThEOREM 5.3. Let $\bar{T}$ be a maximal consistent set of sentences. Let $\alpha$ and $\beta$ be sentences. Then the following hold:

(1) If $\alpha \in \bar{T}$, then $\neg \alpha \notin \bar{T}$.

(2) $\alpha \wedge \beta \in \bar{T}$ iff $\alpha \in \bar{T}$ and $\beta \in \bar{T}$.

(3) If $\bar{T} \vdash \alpha$, then $\alpha \in \bar{T}$, i.e., $\bar{T}$ is deductively closed.

(4) If $\alpha \in \bar{T}$ and $\alpha \rightarrow \beta \in \bar{T}$, then $\beta \in \bar{T}$.

(5) If $P_{\geqslant_{s}} \alpha \in \bar{T}$, and $s \geqslant r$, then $P_{\geqslant_{r}} \alpha \in \bar{T}$.

(6) If $r$ is a rational number and $r=\sup \left\{s: P_{\geqslant_{s}} \alpha \in \bar{T}\right\}$, then $P_{\geqslant_{r}} \alpha \in \bar{T}$. 
Proof. As an example we prove 6. Let $r=\sup \left\{s: P_{\geqslant_{s}} \alpha \in \bar{T}\right\}$. By the inference rule $3, \bar{T} \vdash P_{\geqslant_{r}} \alpha$, and, since $\bar{T}$ is deductively closed set, $P_{\geqslant_{r}} \alpha \in \bar{T}$. The other cases follow similarly.

THEOREM 5.4 (Completeness theorem). Every consistent set $T$ of formulas has an $L C P_{\text {Meas }}$-model.

Proof. Let $T$ be a consistent set of formulas. Let the tuple $\mathbf{M}=(W, v$, Prob $)$ be defined as follows:

- $W$ is the set of all maximal consistent sets,

- $v: W \times I \rightarrow\{$ true, false $\}$ is an assignment such that for every world $w \in W$ and every propositional letter $p \in I, v(w, p)=$ true iff $p \in w$,

- $\operatorname{Prob}(w)=(W(w), H(w), \mu(w))$ is a structure such that:

- $W(w)=W$,

- $H(w)$ is a set of $[\varphi]=\{w \in W: \varphi \in w\}$, for every formula $\varphi$,

$-\mu(w)([\varphi])=\sup \left\{s: P_{\geqslant s}(\varphi) \in w\right\}$.

First we have to prove that $M$ is an $L C P_{\text {Meas }}$-model. For every $w \in W, H(w)$ is an algebra of subsets of $W(w)$. Really, for an arbitrary formula $\alpha, W(w)=[\alpha \mathrm{V}$ $\neg \alpha] \in H(w)$. Also, if $[\alpha] \in H(w)$, then the complement of $[\alpha]$ is the set $[\neg \alpha]$, and it belongs to $H(w)$, and if $\left[\alpha_{1}\right], \ldots\left[\alpha_{n}\right] \in H(w)$, then the union $\left[\alpha_{1}\right] \cup \ldots \cup\left[\alpha_{n}\right] \in H(w)$ because $\left[\alpha_{1}\right] \cup \ldots \cup\left[\alpha_{n}\right]=\left[\alpha_{1} \vee \ldots \vee \alpha_{n}\right]$. Thus, for every $w, H(w)$ is an algebra of subsets of $W(w)$.

Now, we will show that the following hold for all sentences $\alpha$, and $\beta$, and every $w \in W:$

(1) If $[\alpha]=[\beta]$, then $\mu(w)([\alpha])=\mu(w)([\beta])$,

(2) $\mu(w)([\alpha]) \geqslant 0$,

(3) $\mu(w)([\alpha])=1-\mu(w)([\neg \alpha])$, and

(4) $\mu(w)([\alpha] \cup[\beta])=\mu(w)([\alpha])+\mu(w)([\beta])$, for all sentence $\alpha$ and $\beta$ such that $[\alpha] \cap[\beta]=\emptyset$,

i.e. $\mu(w)$ is a finite additive probability measure on $H(w)$.

1. It is enough to prove that $[\alpha] \subset[\beta]$ implies $\mu(w)([\alpha]) \leqslant \mu(w)([\beta])$. From $[\alpha] \subset[\beta]$ it follows that $\vdash \neg(\alpha \wedge \neg \beta)$, and $\vdash P_{\geqslant 1}(\alpha \rightarrow \beta)$. If $P_{\geqslant s} \alpha \in w$, then by Theorem 4.1. 4.1, $P_{\geqslant s} \beta \in w$, and we conclude that $\mu(w)([\alpha]) \leqslant \mu(w)([\beta])$.

2. Since $P_{\geqslant 0} \alpha$ is an axiom, $\mu(w)([\alpha]) \geqslant 0$.

3. Let $r=\mu(w)([\alpha])=\sup \left\{s: P_{\geqslant s} \alpha \in w\right\}$. Suppose that $r=1$. Then, by Theorem 5.3. 6 we have $P_{\geqslant 1} \alpha=P_{\leqslant 0} \neg \alpha=\neg P_{>0} \neg \alpha$, and $\neg P_{>0} \neg \alpha \in w$. If for some $s>0, P \geqslant s \neg \alpha \in w$, by the axiom 3' it must be $P_{>0} \neg \alpha \in w$, a contradiction. It follows that $\mu(w)([\neg \alpha])=1$. Suppose that $r<1$. Then, for every rational number $r^{\prime} \in(r, 1], \neg P_{\geqslant r^{\prime}} \alpha=P_{<r^{\prime}} \alpha$, and $P_{<r^{\prime}} \alpha \in w$. By the axiom $3, P_{\leqslant r^{\prime}} \alpha$ and $P_{\geqslant 1-r^{\prime}}(\neg \alpha)$ belong to $w$. On the other hand, if there is a rational number $r^{\prime \prime} \in[0, r)$ such that $P_{\geqslant 1-r^{\prime \prime}}(\neg \alpha) \in w$, then $\neg P_{>r^{\prime \prime}} \alpha \in w$, a contradiction. Hence, $\sup \left\{s: P_{\geqslant s}(\neg \alpha) \in w\right\}=1-\sup \left\{s: P_{\geqslant s} \alpha \in w\right\}$.

4. Let $[\alpha] \cap[\beta]=\emptyset, \mu(w)([\alpha])=r$ and $\mu(w)([\beta])=s$. Since $[\beta] \subset[\neg \alpha]$, by the step 3, we have $r+s \leqslant r+(1-r)=1$. Suppose that $r>0$, and $s>0$. By the well known properties of the supremum, and monotonicity (Theorem 4.1.4.1) 
for every rational number $r^{\prime} \in[0, r)$, and every rational number $s^{\prime} \in[0, s)$, we have $P_{\geqslant r^{\prime}} \alpha, P_{\geqslant s^{\prime}} \beta \in w$. It follows by the axiom 5 that $P_{\geqslant r^{\prime}+s^{\prime}}(\alpha \vee \beta) \in w$. Hence, $r+s \leqslant \sup \left\{t: P_{\geqslant t}(\alpha \vee \beta) \in w\right\}$. If $r+s=1$, then the assertion trivially holds. Suppose $r+s<1$. If $r+s<t_{0}=\sup \left\{t: P_{\geqslant t}(\alpha \vee \beta) \in w\right\}$, then for every rational number $t^{\prime} \in\left(r+s, t_{0}\right)$ we have $P_{\geqslant t^{\prime}}(\alpha \vee \beta) \in w$. We can choose rational numbers $r^{\prime \prime}>r$ and $s^{\prime \prime}>s$ such that: $\neg P_{\geqslant r^{\prime \prime}} \alpha, P_{<r^{\prime \prime}} \alpha \in w, \neg P_{\geqslant s^{\prime \prime}} \beta, P_{<s^{\prime \prime}}(\beta) \in w$, and $r^{\prime \prime}+s^{\prime \prime}=t^{\prime} \leqslant 1$. By the axiom $4, P_{\leqslant r^{\prime \prime}} \alpha \in w$. Using the axiom 6 we have $P_{<r^{\prime \prime}+s^{\prime \prime}}(\alpha \vee \beta), \neg P_{\geqslant r^{\prime \prime}+s^{\prime \prime}}(\alpha \vee \beta)$, and $\neg P_{\geqslant t^{\prime}}(\alpha \vee \beta) \in w$, a contradiction. Hence, $\mu(w)([\alpha] \cup[\beta])=\mu(w)([\alpha])+\mu(w)([\beta])$. Finally suppose that $r=0$ or $s=0$. Then we can reason as above, with the only exception that $r^{\prime}=0$ or $s^{\prime}=0$.

Finally, by the induction on the complexity of formulas we can prove that for every formula $\alpha$, end every world $w \in W, w \vDash_{\mathbf{M}} \alpha$ iff $\alpha \in w$.

To begin the induction, let $\alpha$ be a propositional letter. Then, $w \vDash_{\mathbf{M}} \alpha$ iff $v(w, \alpha)=$ true iff $\alpha \in w$, by the definition of $v$. Let $\alpha=\neg \beta$. Then $w \vDash_{\mathbf{M}} \neg \beta$ iff $w \forall_{\mathbf{M}} \beta$ iff $\beta \notin w$ iff $\neg \beta \in w$. Let $\alpha=\beta \wedge \gamma . w \vDash_{\mathbf{M}} \beta \wedge \gamma$ iff $w \vDash_{\mathbf{M}} \beta$ and $w \vDash_{\mathbf{M}} \gamma$ iff $\beta \in w$ and $\gamma \in w$ iff $\beta \wedge \gamma \in w$. Let $\alpha=P_{\geqslant_{s}} \beta$. If $\alpha \in w$, $\sup \left\{r: P_{\geqslant r} \beta \in w\right\}=\mu(w)([\beta]) \geqslant s$, and $w \vDash_{\mathbf{M}} P_{\geqslant s} \beta$. For the other direction, suppose that $w \vDash_{\mathbf{M}} P_{\geqslant s} \beta$, i.e., that $\sup \left\{r: P_{\geqslant r} \beta \in w\right\} \geqslant s$. If $\mu(w)([\beta])>s$, then, by the well known property of supremum and monotonicity of $\mu(w), P_{\geqslant s} \beta \in w$. If $\mu(w)([\beta])=s$, then by Theorem 5.3. 6, $P_{\geqslant s} \beta \in w$. Now, let $\alpha=C P_{\leqslant 0}(\beta \mid \gamma)$. If $\alpha \in w$, then, by Axiom 8, $P_{=0}(\beta \wedge \gamma), P_{>0} \gamma \in w$, i.e. $\mu(w)([\beta] \cap[\gamma])=0$ and $\mu(w)([\gamma])>0$. Then, $\frac{\mu(w)([\beta] \cap[\gamma])}{\mu(w)([\gamma])}=0$, and hence $w \vDash_{\mathbf{M}} C P_{\leqslant 0}(\beta \mid \gamma)$. On the other hand, if $w \vDash_{\mathbf{M}} C P_{\leqslant 0}(\beta \mid \gamma)$, i.e. $\frac{\mu(w)([\beta] \cap[\gamma])}{\mu(w)([\gamma])} \leqslant 0$, we have $\mu(w)([\gamma])>0$ and $\mu(w)([\beta] \cap[\gamma])=0$, by Axiom 8. Then, there is $t_{0}>0$ such that $t_{0}=\sup \{t$ : $\left.P_{\geqslant t} \gamma \in w\right\}$, and hence there is rational number $t^{\prime}$ such that $P_{\geqslant t^{\prime}} \gamma \in w$. So, $P_{>0} \gamma \in w$. Also, it is not difficult to Prove that $P_{=0}(\beta \wedge \gamma) \in w$. Then, it follows that $C P_{\leqslant 0}(\beta \mid \gamma) \in w$. At the end, let $\alpha=C P_{\geqslant s}(\beta \mid \gamma)$. Suppose $C P_{\geqslant s}(\beta \mid \gamma) \in w$. We have either $P_{=0} \gamma \in w$ or $P_{=0} \gamma \notin w$. If $P_{=0} \gamma \in w$, then $\mu(w)([\gamma])=0$, and hence $w \vDash_{\mathrm{M}} C P_{\geqslant s}(\beta \mid \gamma)$, for all $s$. If $P_{=0} \gamma \notin w$, then $w \vDash_{\mathrm{M}} C P_{\geqslant s}(\beta \mid \gamma)$ iff $\frac{\mu(w)([\beta] \cap[\gamma])}{\mu(w)([\gamma])} \geqslant s$ iff $\mu(w)([\beta] \cap[\gamma]) \geqslant s \cdot \mu(w)([\gamma])$. Let $t_{0}=\mu(w)([\gamma])>0$. For every $t<t_{0}$ we have $P_{\geqslant t} \gamma \in w$ and $P_{\geqslant t \cdot s}(\beta \wedge \gamma) \in w$ (by Axiom 7 and $\left.C P_{\geqslant s}(\beta \mid \gamma) \in w\right)$. So, $\frac{\mu(w)([\beta] \cap[\gamma])}{\mu(w)([\gamma])} \geqslant s$ and $w \vDash_{\mathbf{M}} C P_{\geqslant s}(\beta \mid \gamma)$. Now, let $w \vDash_{\mathbf{M}} C P_{\geqslant s}(\beta \mid \gamma)$. If $w \vDash_{\mathbf{M}} P_{=0} \gamma$, then $P_{=0} \gamma \in w$, and hence $C P_{=1}(\beta \mid \gamma) \in w$. Since we have $C P_{=1}(\beta \mid \gamma) \rightarrow C P_{\geqslant s}(\beta \mid \gamma)$ for every $s, C P_{\geqslant s}(\beta \mid \gamma) \in w$. If $w \nvdash_{\mathbf{M}} P_{=0} \gamma$, then $\mu(w)([\gamma])=t_{0}>0$. Let $\mu(w)([\beta] \cap[\gamma])=r$. Then we have $\frac{r}{t_{0}} \geqslant s$, i.e. $r \geqslant t_{0} \cdot s$ (since $w \vDash_{\mathbf{M}} C P_{\geqslant s}(\beta \mid \gamma)$, i.e. $\frac{\mu(w)([\beta] \cap[\gamma])}{\mu(w)([\gamma])} \geqslant s$ ). For every rational number $t^{\prime} \leqslant t_{0}$, and every rational number $r^{\prime} \leqslant r$, we have $P_{\geqslant t^{\prime}} \gamma, P_{\geqslant r^{\prime}}(\beta \wedge \gamma) \in w$. Also, for every $t^{\prime}>t_{0}, \neg P_{\geqslant t^{\prime}} \gamma \in w$. So, for every $t^{\prime} \leqslant t_{0}, P_{\geqslant t^{\prime}} \gamma \rightarrow P_{\geqslant t^{\prime} \cdot s}(\beta \wedge \gamma) \in w$ $\left(t^{\prime} \cdot s \leqslant t \cdot s \leqslant r\right)$, and for every $t^{\prime}>t, P_{\geqslant t^{\prime}} \gamma \rightarrow P_{\geqslant t^{\prime} \cdot s}(\beta \wedge \gamma) \in w\left(\right.$ since $\left.\neg P_{\geqslant t^{\prime}} \gamma \in w\right)$. Now, by the inference rule 4 we have $C P_{\geqslant s}(\beta \mid \gamma) \in w$. 


\section{Decidability}

We will use a kind of filtration, as well as linear programming theory to show that the logic $L C P$ is decidable.

TheOREM 6.1. If an LCP-formula $\alpha$ is satisfiable, then it is satisfiable in a finite LCP-model.

Proof. Let $\operatorname{Subfor}(\alpha)$ be the set of all subformulas of $\alpha$. Suppose $\alpha$ holds in a world of an $L C P$-model $M=\langle W, v$, Prob $\rangle$. Let $\approx$ be an equivalence relation over $W^{2}$, such that $w \approx u$ iff $(\forall \theta \in \operatorname{Subfor}(\alpha))\left(w \vDash_{\mathbf{M}} \theta\right.$ iff $\left.u \vDash_{\mathbf{M}} \theta\right)$. The quotient set $W / \approx$ is finite, $W / \approx=\left\{C_{w_{1}}, \ldots, C_{w_{n}}\right\}$ (from every class we choose an element and denoted it by $\left.w_{i}\right)$. We consider a model $M^{*}=\left\langle W^{*}, v^{*}, \operatorname{Prob}^{*}\right\rangle$, where $W^{*}=$ $\left\{w_{1}, \ldots, w_{n}\right\}, v^{*}(w, p)=v(w, p)$, for every propositional letter $p$ and Prob* is defined as follows: $W^{*}\left(w_{i}\right)=\left\{w:\left(\exists u \in C_{w}\right) u \in W\left(w_{i}\right)\right\}, H^{*}\left(w_{i}\right)$ is the power set of $W^{*}\left(w_{i}\right)$, for every $u \in W^{*}, \mu^{*}\left(w_{i}\right)(u)=\mu\left(w_{i}\right)\left(C_{u} \cap W\left(w_{i}\right)\right)$ and for every $D \in H^{*}\left(w_{i}\right), \mu^{*}\left(w_{i}\right)(D)=\sum_{u \in D} \mu\left(w_{i}\right)\left(C_{u} \cap W\left(w_{i}\right)\right)$. Since we have

$\mu^{*}\left(w_{i}\right)\left(W^{*}\left(w_{i}\right)\right)=\sum_{u \in W^{*}\left(w_{i}\right)} \mu\left(w_{i}\right)\left(C_{u} \cap W(w)\right)=\sum_{C u \in W / \approx} \mu\left(w_{i}\right)\left(C_{u} \cap W(w)\right)=1$,

$\mu^{*}\left(w_{i}\right)$ is a probability. It is easy to prove that $M^{*}$ is an $L C P$-model. Now, every formula $\theta \in \operatorname{Subfor}(\alpha)$ is satisfiable in $M$ iff it is satisfiable in $M^{*}$. If $\theta$ is a propositional letter, and $w \vDash_{\mathbf{M}} \theta$, then $w_{i} \vDash_{\mathbf{M}} \theta$ holds for $w_{i} \in C_{w}$. Obviously, $w_{i} \vDash_{\mathbf{M}} \theta$ iff $w_{i} \vDash_{\mathbf{M}^{*}} \theta$. If $\theta=\theta_{1} \wedge \theta_{2}, w \vDash_{\mathbf{M}} \theta$ and $w_{i} \in C_{w}$, then $w_{i} \vDash_{\mathbf{M}} \theta$ iff $w_{i} \vDash_{\mathbf{M}} \theta_{1}$ and $w_{i} \vDash_{\mathbf{M}} \theta_{2}$ iff $w_{i} \vDash_{\mathbf{M}^{*}} \theta_{1}$ and $w_{i} \vDash_{\mathbf{M}^{*}} \theta_{2}$ iff $w_{i} \vDash_{\mathbf{M}^{*}} \theta$. The case $\theta=\neg \varphi$ follows similarly. If $\theta=P_{\geqslant_{s}} \beta$ and $w \vDash_{\mathbf{M}} \theta$, then $w_{i} \vDash_{\mathbf{M}} \theta$ holds for $w_{i} \in C_{w}$, and

$$
\begin{aligned}
w_{i} \vDash_{\mathbf{M}} \theta & \Leftrightarrow \mu\left(w_{i}\right)\left(\left\{u \in W\left(w_{i}\right): u \vDash_{\mathbf{M}} \beta\right\}\right) \geqslant r \\
& \Leftrightarrow \mu\left(w_{i}\right)\left(\bigcup_{u \vDash_{\mathbf{M}} \beta} C_{u} \cap W\left(w_{i}\right)\right) \geqslant r \\
& \Leftrightarrow \mu\left(w_{i}\right)\left(\bigcup_{w_{j} \vDash_{\mathbf{M}} \beta} C_{w_{j}} \cap W\left(w_{i}\right)\right) \geqslant r \\
& \Leftrightarrow \mu\left(w_{i}\right)\left(\bigcup_{w_{j} \vDash_{\mathbf{M}^{*} \beta}} C_{w_{j}} \cap W\left(w_{i}\right)\right) \geqslant r \\
& \Leftrightarrow \mu^{*}\left(w_{i}\right)\left(\left\{w_{j} \in W\left(w_{i}\right): w_{j} \vDash_{\mathbf{M}^{*}} \beta\right\}\right) \geqslant r \\
& \Leftrightarrow w_{i} \vDash_{\mathbf{M}^{*}} \theta .
\end{aligned}
$$

The cases $\theta=C P_{\geqslant s}(\beta \mid \gamma)$ and $\theta=C P_{\leqslant 0}(\beta \mid \gamma)$ follow similarly.

The model $\mathbf{M}^{*}$ from the theorem has no more then $2^{n}$ worlds, where $n$ is a number of subformulas of the considered formula $\alpha$.

TheOrem 6.2. The logic LCP is decidable. 
Proof. Suppose that $\alpha$ holds in a world $w$ of an model $M=(W, v$, Prob). Let

$$
\operatorname{Subfor}(\alpha)=\left\{\varphi_{1}, \ldots, \varphi_{k}\right\}
$$

be the set of all subformulas of $\alpha$. In each world holds exactly one conjunction of the form $\pm \varphi_{1} \wedge \ldots \wedge \pm \varphi_{k}$, called characteristic formula of that world. Let $\alpha_{1}, \ldots, \alpha_{2^{k}}$ be the list of all characteristic formulas. By Theorem 6.1, there is an $L C P$-model of cardinality $\leqslant 2^{k}$ and $\alpha$ is satisfiable in at least one world from the model. For every natural number $l \leqslant 2^{k}$, we consider models which have $l$ worlds. In each of these worlds holds exactly one characteristic formula. So, for every $l$ we consider all sets containing $l$ characteristic formulas, which are propositional consistent and at least one contains $\alpha$. For every such choice and each world $w_{i}$ (i.e. the corresponding characteristic formula $\left.\alpha_{i}\right)$ let $X_{r_{1}} \beta_{r_{1}}, \ldots, X_{r_{p}} \beta_{r_{p}}, Y_{s_{1}}\left(\gamma_{1} \mid \delta_{1}\right), \ldots, Y_{s_{q}}\left(\gamma_{q} \mid \delta_{q}\right)$, $C P_{\leqslant 0}\left(\eta_{1} \mid \theta_{1}\right), \ldots, C P_{\leqslant 0}\left(\eta_{t} \mid \theta_{t}\right), p+q+t \leqslant k$, be an enumeration of all probability formulas which appear as conjuncts in $\alpha_{i}$, where $X_{r}$ is a probability operator from the set $\left\{P_{\geqslant r}, P_{<r}\right\}$ and $Y_{s}$ is a probability operator from the set $\left\{C P_{\geqslant s}, C P_{<s}\right\}$. We consider the following systems of linear equalities and inequalities with unknowns $x_{j}^{i}$ (denoting $\left.\mu\left(w_{i}\right)\left(w_{j}\right)\right), i=0,1, \ldots, l, j=1,2, \ldots, l$ (we use expression of the form $\varphi \in \alpha_{i}$ to denote that the formula $\varphi$ appears as a conjunct in $\alpha_{i}$ ):

$$
\begin{aligned}
& \sum_{j=1}^{l} x_{j}^{i}=1 ; x_{j}^{i} \geqslant 0(i=1, \ldots, l), \\
& \sum_{j: \beta \in \alpha_{j}} x_{j}^{i} \geqslant r_{k}, \text { if } P_{\geqslant r_{k}} \beta \in \alpha_{i} ; \\
& \sum_{j: \beta \in \alpha_{j}} x_{j}^{i}<r_{k}, \text { if } P_{<r_{k}} \beta \in \alpha_{i} ; \\
& \sum_{j: \gamma \wedge \delta \in \alpha_{j}} x_{j}^{i} \geqslant s \sum_{j: \gamma \in \alpha_{j}} x_{j}^{i} \text { and } \sum_{j: \gamma \in \alpha_{j}} x_{j}^{i}>0, \\
& \text { or } \sum_{j: \gamma \in \alpha_{j}} x_{j}^{i}=0, \text { if } C P_{\geqslant s}(\gamma \mid \delta) \in \alpha_{i} ; \\
& \sum_{j: \gamma \wedge \delta \in \alpha_{j}} x_{j}^{i}<s \sum_{j: \gamma \in \alpha_{j}} x_{j}^{i} \text { and } \sum_{j: \gamma \in \alpha_{j}} x_{j}^{i}>0, \text { if } C P_{<s}(\gamma \mid \delta) \in \alpha_{i} ; \\
& \sum_{j: \eta \wedge \theta \in \alpha_{j}} x_{j}^{i}=0, \sum_{j: \theta \in \alpha_{j}} x_{j}^{i}>0, \text { if } C P_{\leqslant 0}(\eta \mid \theta) \in \alpha_{i} .
\end{aligned}
$$

Since the problem of satisfiability of $\alpha$ is reduced to the linear system solving problem, the $L C P$ is decidable.

\section{Conclusions}

In this paper we have presented a probability logic which is suitable for reasoning about higher-order conditional probability in the sense of Kolmogorov. It might be applied, for example, to consider Miller's principle, a well-known principle relating higher order probabilities. Among a number of variants of Miller's principle, one of most interesting can be expressed in $L C P$ by the formula $C P_{\geqslant_{s}}\left(\varphi \mid P_{\geqslant_{s}} \varphi\right)$. Since Miller's principle is not sound with respect to the class of $L C P$-models, it would be interesting to characterize structures satisfying it.

There are some papers that consider conditional probabilities from the logical point. Hawthorne [4] described a range of nonmonotonic conditional that behave like conditional probability functions at various levels of probabilistic supports. These conditional were defined as semantic relation on an object language for propositional logic. In [5] he extended the semantics of the most prominent family of these nonmonotonic conditionals to a language for predicate logic. In [2] 
conditional probability is defined syntactically. However, a complicated machinery of real closed fields is needed to obtain the corresponding sound and complete axiomatization. In $[\mathbf{1 6}, \mathbf{1 7}]$ a logic, which contains several types of probabilistic operators (including operators of the form "the conditional probability of $\alpha$ given $\beta$ is $s$ ), is defined. The range of the probability functions is taken to be the unit interval of a recursive nonarchimedean field. Thus, it is possible to define another probabilistic operator with the intended meaning "probabilities of $\alpha \wedge \beta$ and $\beta$ are almost the same" which may be used to model default reasoning. In $[\mathbf{3}]$ a treatment of nonstandard conditional probability by means of fuzzy logic is given. Using de Finetti's approaches to conditional probability, a fuzzy modal logic is introduced in [7], such that for each pair of classical propositional formulas $\alpha$ and $\beta$, the probability of the conditional event " $\alpha$ given $\beta$ " is taken as the truth-value of the (fuzzy) modal proposition $P(\alpha \mid \beta)$. One of the interesting problems might be to find axiomatization of the logic with higher order conditional probability operators of de Finetti's type. Namely, allowing iterations of probability operators can help us to formalize uncertainty of probabilities. Another direction for further research might be extending our logic to corresponding first order logics. All these formalizations can be seen as useful tool in modelling and understanding real-world problems.

\section{References}

[1] S. Coletti, R. Scozzafava, Probabilistic logic in a coherent setting, Kluwer, Dordrecht, 2002.

[2] R. Fagin, J. Halpern, N. Megiddo, A logic for reasoning about probabilities, Information and Computation 87:1/2 (1990), 78-128.

[3] T. Flaminio, F. Montagna, A logical and algebraic treatment of conditional probability; in: L. A. Zadeh (ed.) Proceedings of IPMU '04, Perugia, Italy, 2004, 493-500.

[4] J. Hawthorne, On the logic of nonmonotonic conditionals and conditional probabilities, J. Philos. Logic 25:1 (v), 185-218.

[5] J. Hawthorne, On the logic of nonmonotonic conditionals and conditional probabilities: Predicate logic, J. Philos. Logic 27:1 (1998), 1-34.

[6] N. Ikodinović, Z. Ognjanović, A logic with coherent conditional probabilities, Proceedings of the 8th European Conference Symbolic and Quantitative Approaches to Reasoning with Uncertainty ECSQARU-2005, Barcelona, Spain, July 6-9, 2005 editor Luis Godo, Lecture Notes in Computer Science Vol. 3571 726-736, 2005.

[7] E. Marchioni, L. Godo, A Logic for Reasoning about Coherent Conditional Probability: A Modal Fuzzy Logic Approach, JELIA 2004: 213-225.

[8] Z. Marković, Z. Ognjanović, M. Rašković, A Probabilistic Extension of Intuitionistic Logic, Math. Logic Quart. 49:5 (2003), 415-424.

[9] N. Nilsson, Probabilistic logic, Artificial Inteligence 28 (1986), 71-87.

[10] N. Nilsson, Probabilistic logic revised, Artificial inteligence 59 (1993), 39-42.

[11] Z. Ognjanović, M. Rašković, A logic with higher order probabilities, Publ. Inst. Math. Nouv. Sér. 60(74) (1996), 1-4.

[12] Z. Ognjanović, M. Rašković, Some probability logics with new types of probability operators, J. Logic Comput. 9:2 (1999), 181-195.

[13] Z. Ognjanović, M. Rašković, Some first order probability logics, Theoret. Comput. Sci. 247:1-2 (2000), 191-212.

[14] M. Rašković, Classical logic with some probability operators, Publ. Inst. Math. Nouv. Sér. 53(67) (1993), 1-3.

[15] M. Rašković, Z. Ognjanović, A first order probability logic $-L P_{Q}$, Publ. Inst. Math. Nouv. Sér. 65(79) (1999), 1-7. 
[16] M. Rašković, Z. Ognjanović, Z. Marković, A probabilistic Approach to Default Reasoning, 10th International Workshop on Non-Monotonic Reasoning NMR2004, Westin Whistler, Canada, June 6-8, 335-341, 2004.

[17] M. Rašković, Z. Ognjanović, Z. Marković, A Logic with Conditional Probabilities, 9th Euroean conference JELIA'04 Logics in Artificial Inteligence, Lecture notes in artificial inteligence (LNCS/LNAI) 3229, 226-238, 2004.

Matematički institut

Kneza Mihaila 36

11000 Beograd, p.p. 367

Serbia

zorano@mi.sanu.ac.yu

Prirodno-matematički fakultet

34000 Kragujevac

Serbia

ikodinovic@kg.ac . yu 